\title{
Quantum and semiclassical Husimi distributions for a one-dimensional resonant system
}

\author{
R. Egydio de Carvalho \\ Instituto de Geociências e Ciências Exatas, Universidade Estadual Paulista, Rio Claro, 13500 São Paulo, Brazil \\ M. A. M. de Aguiar \\ Instituto de Física "Gleb Wataghin," Universidade Estadual de Campinas, Campinas, 13081 São Paulo, Brazil
}

(Received 13 November 1991)

\begin{abstract}
We compare exact and semiclassical Husimi distributions for the single eigenstates of a onedimensional resonant Hamiltonian. We find that both distributions concentrate near the unstable fixed points even when these points are made complex by suitably varying a parameter.
\end{abstract}

PACS number(s): 05.45. $+\mathrm{b}, 03.20 .+\mathrm{i}$

The study of the semiclassical limit of quantum mechanics is as old as quantum theory itself. Comparing the features of both theories is, however, not a trivial task, due to the very different languages that they employ. In order to overcome this difficulty, Wigner [1] in 1932 provided a rule to associate to every quantum state $\psi(q)$ a phase-space function $W_{\psi}(q, p)$ through

$$
\begin{gathered}
W_{\psi}(q, p)=\frac{1}{2 \pi \hbar} \int_{-\infty}^{+\infty} d x \psi^{*}(q+x / 2) \psi(q-x / 2) \\
\times e^{i p x / \hbar} .
\end{gathered}
$$

Although $W$ is real valued and projects onto the correct marginal distributions, it is necessarily negative in some regions of phase space [2]. This implies that the Wigner function cannot be interpreted as a probability density. In practice, simple examples show that $W(q, p)$ oscillates violently in the semiclassical limit [3], suggesting that these oscillations could be washed out be a suitable averaging. Indeed, Husimi [4] showed that a Gaussian smoothing of (1) would result in a positive quantity. The Husimi function for the eigenstate $\psi_{m}$ is defined by

$$
\begin{aligned}
h_{m}(q, p)= & \frac{1}{\pi \hbar} \int d q^{\prime} d p^{\prime} W_{m}\left(q^{\prime}, p^{\prime}\right) \\
& \times \exp \left\{-\left(q-q^{\prime}\right)^{2} / 2 b^{2}-\left(p-p^{\prime}\right)^{2} b^{2} / 2 \hbar^{2}\right\} \\
= & \left|\left\langle z \mid \psi_{m}\right\rangle\right|^{2}
\end{aligned}
$$

where $|z\rangle$ is the coherent state for the harmonic oscillator,

$$
z=(1 / \sqrt{2})(q / b+i p b / \hbar),
$$

and $b$ is a free parameter controlling the Gaussian width.

Semiclassical expressions for both Wigner [4,5] and Husimi [6] functions have been obtained recently but not yet tested numerically.

For one degree of freedom the semiclassical limit of (2) is given by [6]

$$
\tilde{h}_{m}(q, p)=\frac{\sqrt{2 \pi \hbar}}{|\dot{z}| \tau\left(E_{m}\right)} \exp \left\{-\frac{\left(E_{m}-E\right)^{2}}{2 \hbar|\dot{z}|^{2}}\right\},
$$

where $E_{m}$ is the eigenenergy, $\tau\left(E_{m}\right)$ is the period of the classical orbit with energy $E_{m}, E=H(q, p)$ and the overdot means time derivative. The two important features of (4) are the Gaussian concentration on the energy shell and the dependence on $|\dot{z}|$, showing that the fixed points play an important role in $\widetilde{h}_{m}(q, p)$.

The purpose of this paper is to compare exact and semiclassical Husimi distributions for a one-dimensional integrable Hamiltonian system exhibiting two resonances. Although simple, this model presents a complex tori structure where the fixed points determine that certain regions of phase space are more frequently visited by the orbits. Moreover, as a parameter is varied, one of the resonances disappears. As we shall see, this model constitutes a very interesting example to check the validity of the semiclassical approximation for the Husimi distributions.

We start by considering an autonomous classical Hamiltonian system with two degrees of freedom in actionangle variable $(J, \theta)$ given by the truncated BirkhoffGustavson normal form $[7,8]$ :

$$
H(\mathbf{J}, \boldsymbol{\theta})=H_{0}(\mathbf{J})+\alpha H_{1}\left(\mathbf{J}, \theta_{1}\right),
$$

where

$$
\begin{aligned}
& H_{0}=J_{2}+6 \lambda J_{1}-c\left(6 J_{1}\right)^{2}+a\left(6 J_{1}\right)^{3}, \\
& H_{1}=\left(6 J_{1}\right)^{3}\left(J_{2}-J_{1}\right)^{1 / 2} \cos \theta_{1},
\end{aligned}
$$

and $\lambda, c, a$, and $\alpha$ are parameters.

As $J_{2}$ is conserved, we concentrate our analysis on the $J_{1}-\theta_{1}$ plane. It is easy to check that the origin $J_{1}=0$ corresponds to a stable periodic orbit. The cubic dependence of $H_{0}$ on $J_{1}$ and the term $\cos \theta_{1}$ in $H_{1}$ produces two separate resonances (two islands) of the same order, with unstable periodic orbits at $\theta_{1}=0$ for one resonance and $\theta_{1}=\pi$ for the other. As the parameter $\alpha$ is varied, the hyperbolic point at $\theta_{1}=0$ vanishes through a saddlecenter bifurcation and a single resonance remains. In what follows we shall fix the parameters values as $\lambda=1 / 32.1, c=6^{-3} / 4, a=6^{-6} / 2$, and $J_{2}=29.765$.

By computational convenience we analyze the motion in the Cartesian coordinates $(p, q)$ connected with $\left(J_{1}, \theta_{1}\right)$ by the canonical transformation,

$$
q=\sqrt{2 J_{1}} \cos \theta_{1}, \quad p=\sqrt{2 J_{1}} \sin \theta_{1} .
$$




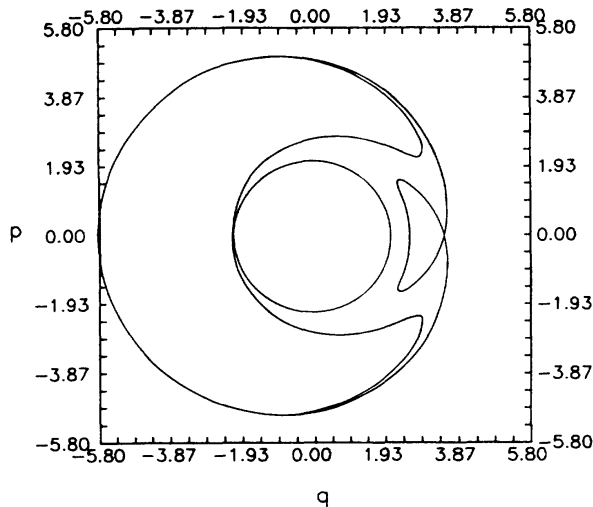

FIG. 1. Separatrices of the two unstable fixed points in the $(q, p)$ plane for $\alpha=1.1 \times 6^{-8}$.

This transformation curves the space $\left(J_{1}, \theta_{1}\right)$ attaching the point $\theta_{1}=0$ with $\theta_{1}=2 \pi$. Figures 1 and 2 show the separatrices for the cases $\alpha=1.1 \times 6^{-8}$ and $1.3 \times 6^{-8}$, respectively.

To calculate the semiclassical and quantal Husimi distributions we need to know the eigenvalues and eigenfunctions of the corresponding Hamiltonian operator. The quantization of the normal form [9] is better visualized when described in terms of the complex variables $\left(\mathbf{a}, \mathbf{a}^{*}\right)$ defined by

$$
\mathbf{a}=\sqrt{\mathbf{I}} \exp (i \varphi), \quad \mathbf{a}^{*}=\sqrt{\operatorname{I}} \exp (-i \varphi),
$$

where $(\mathbf{I}, \boldsymbol{\varphi})$ are related to $(\mathbf{J}, \boldsymbol{\theta})$ by canonical transformation

$J_{1}=\frac{I_{1}}{6}, \theta_{1}=6 \varphi_{1}-\varphi_{2}, \quad J_{2}=I_{2}+\frac{I_{1}}{6}, \theta_{2}=\varphi_{2}$.

The quantization of $\left(\mathbf{a}, \mathbf{a}^{*}\right)$ defines the annihilation and

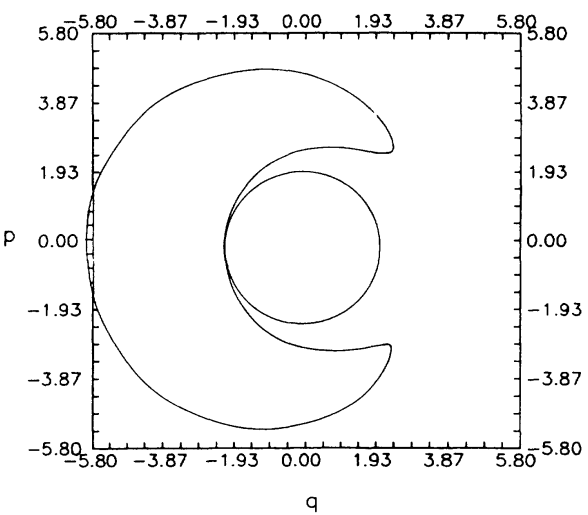

FIG. 2. Separatrix of the only remaining unstable fixed point in the $(q, p)$ plane for $\alpha=1.3 \times 6^{-8}$.

creation operators, respectively, where $\left[\widehat{a}_{j}, \widehat{a}_{k}^{\dagger}\right]=\hbar \delta_{j, k}$. The operator $\widehat{J}_{2}$ is also a constant of motion, so that a base of eigenstates of $\hat{J}_{2}$ puts the Hamiltonian matrix in a block form. To get these eigenstates we first define a basis of harmonic oscillators for the operators $\hat{I}_{j}=\left(a_{j}^{\dagger} a_{j}+\frac{1}{2}\right), \quad\left|n_{1}, n_{2}\right\rangle$, so that $\hat{I}_{j}\left|n_{1}, n_{2}\right\rangle=\hbar\left(n_{j}\right.$ $\left.+\frac{1}{2}\right)\left|n_{1}, n_{2}\right\rangle$. Then, in analogy to Eqs. (8) we define the operator $\hat{J}_{1}$ and $\hat{J}_{2}$ which, acting on $\left|n_{1}, n_{2}\right\rangle$, define the quantum numbers $(n, m)$ with $n_{1}=6 n+n_{0}$ and $n_{2}=m-n$ where $n_{0}=0,1,2,3,4$, or 5 fixes one of the sixfold symmetry blocks. In this paper we have chosen $n_{0}=0$. Therefore, we define the basis vector

$$
|k\rangle \equiv\left|6 n+n_{0}, m-n\right\rangle
$$

and for each fixed value of $m$ we obtain a block of the Hamiltonian matrix. The matrix elements are then given by

$$
\begin{aligned}
\left\langle k^{\prime}|\hat{H}| k\right\rangle= & \left\{\hbar\left[m+\frac{13}{12}\right]+\lambda \hbar\left[6 n+\frac{7}{2}\right]-c \hbar^{2}\left[6 n+\frac{7}{2}\right]^{2}+a \hbar^{3}\left[6 n+\frac{7}{2}\right]^{3}\right\} \delta_{n^{\prime}, n} \delta_{m^{\prime}, m} \\
& +\frac{\alpha}{2} \hbar^{7 / 2}\{(6 n+4)(6 n+5)(6 n+6)(6 n+7)(6 n+8)(6 n+9)(m-n)\}^{1 / 2} \delta_{n^{\prime}, n+1} \delta_{m^{\prime}, m}+\text { H.c. }
\end{aligned}
$$

where H.c. means Hermitian complex term.

The integrable resonant perturbation implies that the Hamiltonian matrix is not only real and symmetric but also separated by uncoupled blocks, where each block is tridiagonal and finite. Therefore the system is reduced to one degree of freedom and we interpret the classical Hamiltonian Eq. (5) as a truly one-dimensional system where $J_{2}$ is just a parameter and every orbit is periodic. The block which corresponds to the value used classically for $J_{2}$ has dimensions $(179 \times 179)$ and the eigenvalues and eigenvectors of $H_{k^{\prime}, k}$ are obtained by direct diagonalization of the matrix.

Let $\left|\psi_{n}\right\rangle, n=0,1, \ldots, m$ be the eigenfunctions of the block labeled by $m$. In terms of the basis vectors $|k\rangle$

$$
\left|\psi_{n}\right\rangle=\sum_{k=0}^{m} c_{k}|k\rangle \text {. }
$$

Therefore, from the definition (2), the Husimi distribution reads

$$
\begin{aligned}
& h_{n}(q, p)=\left|\left\langle z \mid \psi_{n}\right\rangle\right|^{2} \\
& \quad=\left|\sum_{k=0}^{m} c_{k}\langle z \mid k\rangle\right|^{2}=\left|\sum_{k=0}^{m} c_{k} \frac{z^{k} e^{-z \bar{z} / 2 \hbar}}{\sqrt{\hbar^{k} k !}}\right|^{2}, \\
& z=(1 / \sqrt{2 \hbar})(q+i p) .
\end{aligned}
$$

The Gaussian width $b$ has been chosen as $\sqrt{\hbar}$ to fit the harmonic-oscillator term of the Hamiltonian in terms of $I$ and $\varphi$. 

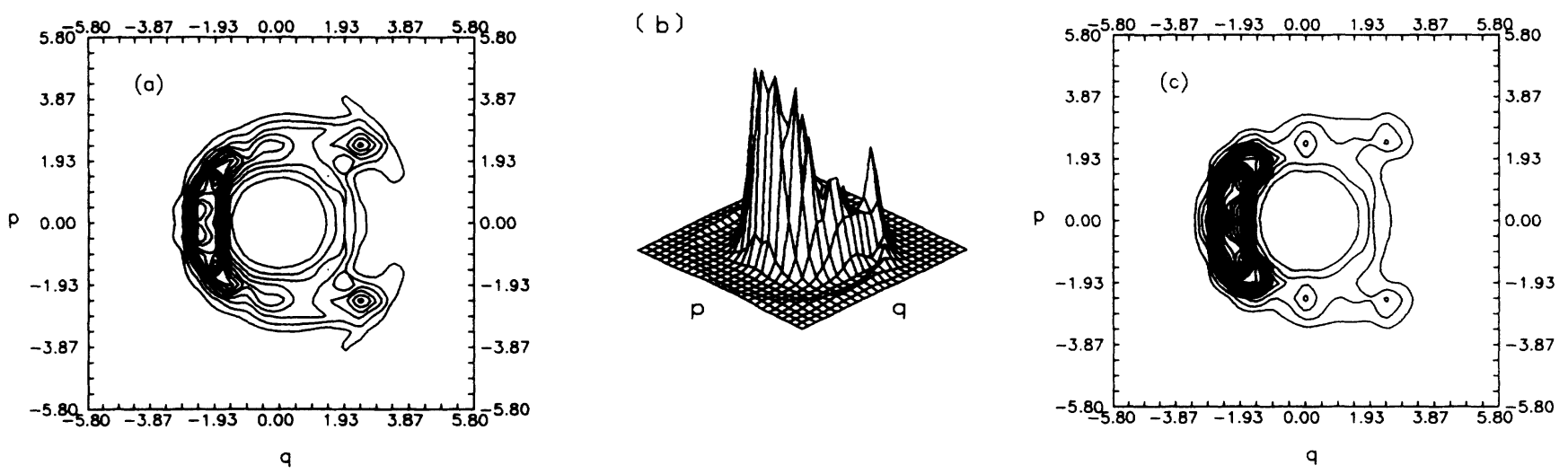

FIG. 3. Husimi distribution for state 58, $\hbar=\frac{1}{6}$, and $\alpha=1.1 \times 6^{-8}$. (a) shows contour plots of the semiclassical calculation [Eq. (4)], with level curves from 0 to 0.096 , in steps of 0.008 . (b) shows the three-dimensional plot and (c) shows contour plots for the exact distribution [Eq. (12)], with level curves from 0 to 0.152 , in steps of 0.008 .
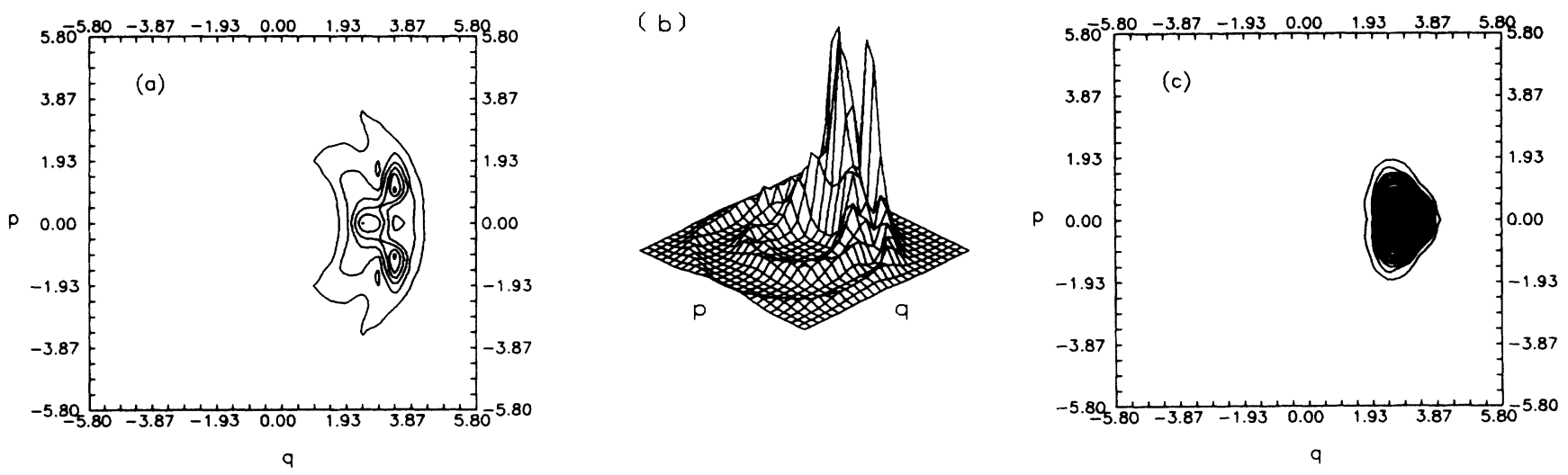

FIG. 4. The same as in Fig. 3 for state 72 and $\alpha=1.1 \times 6^{-8}$. In (a) the curves go from 0 to 0.12 , in steps of 0.02 and in (c) from 0 to 0.52 , in steps of 0.02 .
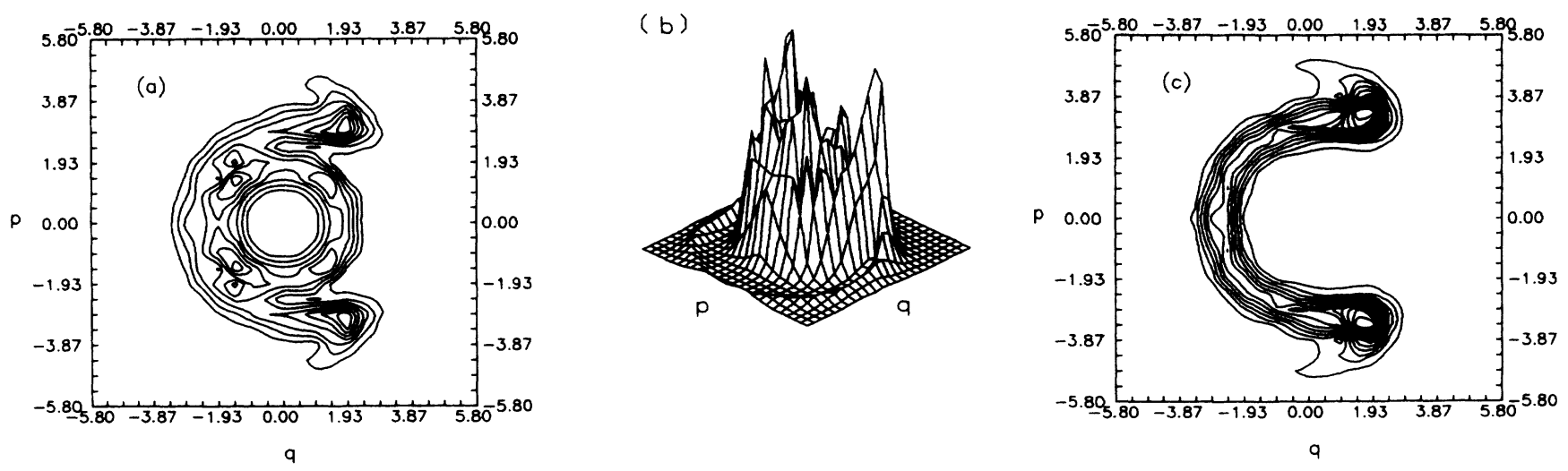

FIG. 5. The same as in Fig. 3 for state 51 and $\alpha=1.3 \times 6^{-8}$. In (a) the curves go from 0 to 0.056 , in steps of 0.008 and in (c) from 0 to 0.096 , in steps of 0.008 . 

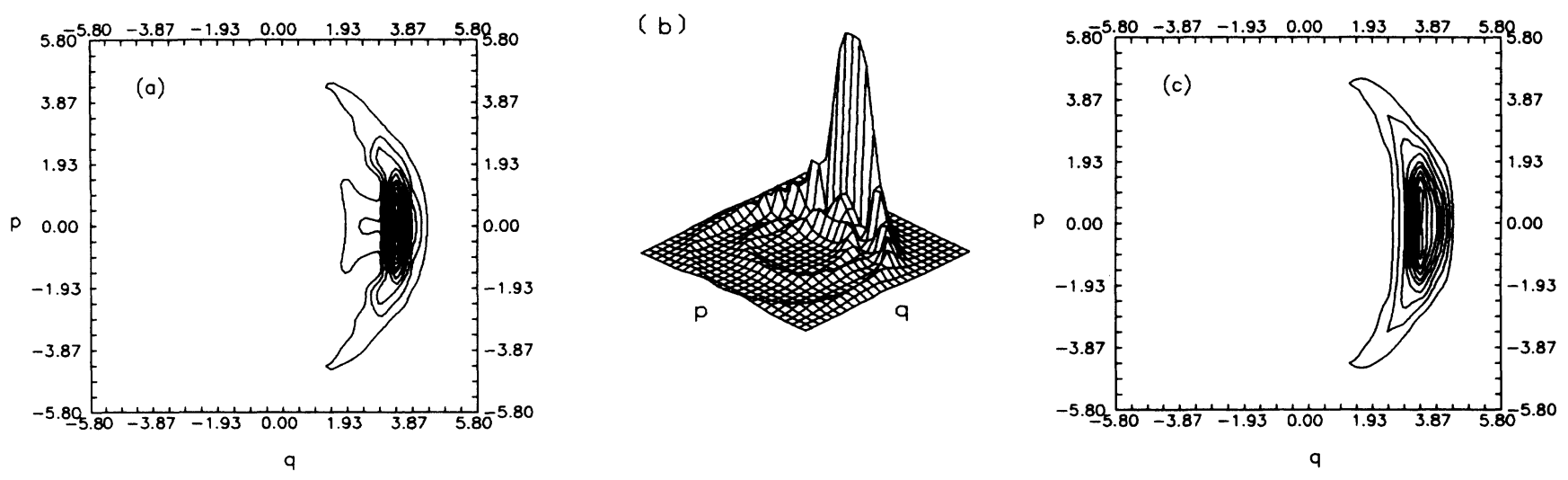

FIG. 6. The same as in Fig. 3 for state 74 and $\alpha=1.3 \times 6^{-8}$. In (a) the curves go from 0 to 0.22 , in steps of 0.02 and in (c) from 0 to 0.18 , in steps of 0.02 .

Figures 3-6 show examples of the exact Husimi distribution [Eq. (12)] and their semiclassical counterparts [Eq. (4)]. In Figs. 3 and $4, \alpha=1.1 \times 6^{-8}$, corresponding to the classical situation described in Fig. 1, while for Figs. 5 and 6, $\alpha=1.3 \times 6^{-8}$, corresponding to Fig. 2. These eigenstates were selected for having energies close to the separatrices in each case. Both classical and quantal distributions have been normalized so as to have unit integral over $q$ and $p$. In all contour plots the level curves go from zero to the maximum value reached by $h(q, p)$ at constant step (see captions).

Besides the very good agreement between the distributions we notice that most semiclassical Husimi distributions are more spread when compared to the quantum ones, an effect similar to the scarring phenomena of chaotic systems. This is also verified in the behavior of neighboring states: some are more uniformly distributed along the energy shell and some exhibit concentrations like the states shown in the figures. Another effect is, however, more important in our results: although for $\alpha=1.3 \times 6^{-8}$ the fixed point corresponding to $\theta_{1}=0$ has vanished from the real plane and has become complex, it still has a very strong influence on the wave functions, as can be seen by the peaked distribution of Fig. 6 around this point. The scar of the complex point also appears in several neighboring states. This fact suggests that complex periodic orbits might play a very important role in the theory of scars for higher-dimensional systems.

We thank A.M. Ozorio de Almeida for interesting discussions. This work was partly supported by FAPESP, CNPq, and FINEP.
[1] E. P. Wigner, Phys. Rev. 40, 749 (1932).

[2] M. Hillery et al., Phys. Rep. 106, 121 (1984).

[3] A. M. Ozorio de Almeida, Hamiltonian Systems: Chaos and Quantization (Cambridge University Press, Cambridge, 1989).

[4] K. Husimi, Proc. Phys. Math. Soc. Jpn. 22, 264 (1940); K. Takahashi, Jpn. 55, 1443 (1986).

[5] M. V. Berry, Proc. R. Soc. London Ser. A 423, 219 (1989).

[6] M. Baranger and M. A. M. de Aguiar (unpublished); J.
Kurchan et al., Phys. Rev. A 40, 6800 (1989).

[7] F. G. Gustavson, Astron. J. 71, (1966) 670.

[8] R. Egydio de Carvalho, Ph.D. thesis, UNICAMP, 1989; R. Egydio de Carvalho and A. M. Ozorio de Almeida, Phys. Lett. A 162, 457 (1992).

[9] M. Robnik, J. Phys. A 17, 109 (1984); R. Egydio de Carvalho and A. M. Ozorio de Almeida, Rev. Bras. Fis. 18, 400 (1988). 